\title{
Feedforward Mechanisms of Excitatory and Inhibitory Cortical Receptive Fields
}

\author{
Randy M. Bruno and Daniel J. Simons \\ Department of Neurobiology, University of Pittsburgh School of Medicine, Pittsburgh, Pennsylvania 15261
}

\begin{abstract}
Excitatory and inhibitory cortical layer IV neurons have distinctive response properties. Thalamocortical connectivity that may underlie differences was examined using cross-correlation analyses of pairs of thalamic and cortical neurons in the rat whisker/barrel system. Cortical layer IV cells discharging fast spikes, presumed inhibitory neurons, were distinguished from regular-spike units, presumed excitatory neurons, by the extracellular waveform shape. Regular-spike neurons fired less robustly and had smaller receptive fields (RFs) and greater directional tuning than fast-spike cells. Presumed excitatory neurons were less likely to receive thalamocortical connections, and their connections were, on average, weaker. RF properties of thalamic inputs to both cell types were equivalent, except that
\end{abstract}

the most highly responsive thalamic cells contacted only fastspike neurons. In contrast, the size and directional tuning of cortical RFs were related to the number of detectable thalamocortical inputs. Connected thalamocortical pairs were likely to have matching RF characteristics. The smaller, more directionally selective RFs of excitatory neurons may be a consequence of their weaker net thalamic drive, their more nonlinear firing characteristics and pervasive feedforward inhibition provided by strongly driven, broadly tuned inhibitory neurons.

Key words: thalamocortical; cortical circuitry; whisker; barrel; barreloid; ventroposterior medial nucleus; thalamus; cross correlation
Studies of the mammalian visual system suggest that receptive fields (RFs) of cortical layer IV neurons are strongly determined by their thalamocortical inputs (Hubel and Wiesel, 1962; Tanaka, 1985; Reid and Alonso, 1995; Ferster et al., 1996). Experiments in the rat somatosensory system have shown that excitatory and inhibitory cortical cells have different RF properties, and that such differences may be similarly explained by feedforward mechanisms (Simons and Carvell, 1989). In whisker-related cortical barrels, inhibitory cells are driven by deflections of the "principal" whisker (PW) of the barrel and several adjacent whiskers (AWs), yet many excitatory cells respond to the PW alone; RFs of thalamocortical neurons vary in size. The multi-whisker RFs of inhibitory cells may reflect the combination of single- and multiwhisker thalamic inputs, whereas the single-whisker RFs of excitatory cells may reflect the commonality of similarly diverse inputs. An explicit assumption is that inhibitory and excitatory cells receive inputs from the same pool of thalamocortical neurons but process them differently.

In addition to their larger RFs, inhibitory neurons have higher spontaneous and evoked firing rates and less directional selectivity (Simons and Carvell, 1989; Armstrong-James et al., 1993; Kyriazi et al., 1994). Excitatory and inhibitory barrel neurons possess different biophysical properties that can readily account for differences in their overall firing rates (Kawaguchi, 1993; Angulo et al., 1999). Differences in the strength and/or frequency

\footnotetext{
Received July 12, 2002; revised Sept. 27, 2002; accepted Oct. 1, 2002.

This work was supported by National Institutes of Health Grant NS19950. We thank H. Kyriazi for expert technical assistance, V. Ventura for advice on implementing the bootstrap, and A. Myers for histology. D. Gillespie, K. Kandler, A. Keller, V. Khatri, H. Kyriazi, and S. Temereanca provided valuable comments on this manuscript.

Correspondence should be addressed to Dr. Randy M. Bruno, Department of Neurobiology, University of Pittsburgh School of Medicine, 3500 Terrace Street, Pittsburgh, PA 15261. E-mail: rbruno@pitt.edu.

Copyright (C) 2002 Society for Neuroscience $0270-6474 / 02 / 2210966-10 \$ 15.00 / 0$
}

of thalamocortical connections may underlie the distinctive RF properties of excitatory and inhibitory cells, but such details of thalamocortical connectivity have not been examined. Also, it is unknown whether the two cell types receive inputs from similar thalamic populations.

Rapid action potentials are discharged by some types of inhibitory neurons. Fast-spike units (FSUs) were first described in extracellular recordings from monkey somatosensory cortex as "thin spikes" (Mountcastle et al., 1969) and later in rat barrel cortex as "fast spikes" (Simons, 1978). Available evidence indicates that FSUs in barrels, although not necessarily elsewhere (Gray and McCormick, 1996), correspond to a prominent subclass of inhibitory neurons (Kawaguchi and Kubota, 1993; Gibson et al., 1999; Rudy et al., 1999; Porter et al., 2001). Slower action potentials, called "regular spikes," are discharged by excitatory, spiny cells, which compose $\sim 90 \%$ of the cortical population (Beaulieu, 1993); regular spikes are also discharged by a sparse subpopulation of GABAergic neurons (Kawaguchi and Kubota, 1993; Gibson et al., 1999).

The present study in the rat whisker/barrel system uses quantitative RF analyses to investigate the relationships between connectivity and response properties of paired thalamic and cortical cells. Regular-spike units (RSUs), presumed excitatory neurons, fired less robustly and had smaller RFs and sharper directional tuning than FSUs, presumed inhibitory cells. Thalamic convergence onto topographically aligned RSUs was twofold less than onto FSUs, and contacts onto RSUs were less efficacious. RF properties of thalamic inputs to both cell types were equivalent, except that the most highly responsive thalamic cells contacted only FSUs. Thus, compared with inhibitory barrel neurons, excitatory cells receive less net thalamic input, rendering them highly susceptible to the effects of strong feedforward inhibition. 


\section{MATERIALS AND METHODS}

Thirty-six adult female rats weighing 200-375 gm (Sprague Dawley strain; Hill Top Lab Animals, Scottsdale, PA) were used in this study. Procedural details have been presented previously (Simons and Carvell, 1989). Briefly, rats were initially anesthetized with halothane (1.5-2.0\%). Bone overlying the right barrel cortex $\left(<0.4 \mathrm{~mm}^{2}\right)$ and ventroposterior medial nucleus (VPM) of the thalamus $\left(<2 \mathrm{~mm}^{2}\right)$ was removed. The body temperature was maintained at $37^{\circ} \mathrm{C}$ by a servo-controlled heating blanket (Harvard Apparatus, Holliston, MA). For neural recordings, halothane was discontinued and the rat was maintained in a lightly narcotized, sedated state by intravenous infusion of fentanyl (Sublimaze, $\sim 10 \mu \mathrm{g} \cdot \mathrm{kg}^{-1} \cdot \mathrm{hr}^{-1}$; Janssen Biochimica, Berse, Belgium). To prevent spontaneous whisker movement, neuromuscular blockade was induced with pancuronium bromide $\left(1.6 \mathrm{mg} \cdot \mathrm{kg}^{-1} \cdot \mathrm{hr}^{-1}\right)$, and the animal was put on artificial respiration ( $\sim 90$ breaths/min) using a positive-pressure ventilator. A Macintosh computer continuously monitored the rat's electroencephalogram, mean arterial pressure, arterial pulse rate, and tracheal airway pressure. Experiments were terminated if any of the above indicators could not be maintained within the normal physiological range.

Extracellular single-unit recordings were obtained using highimpedance (5-12 M $\Omega$ ) electrodes made from pulled and beveled $90 \%$ quartz-insulated platinum and 10\% tungsten core fibers (Uwe Thomas Recording, Giessen, Germany). In our hands, these electrodes are wellsuited for isolating FSUs. Electrodes were slowly advanced perpendicular to the pial surface using either a hydraulic microdrive (David Kopf Instruments, Tujunga, CA) or an Eckhorn microdrive (Uwe Thomas Recording). Signals were amplified by conventional means and bandpassfiltered $300-10 \mathrm{kHz}$; the high setting of the low-pass filter is essential for capturing the distinctive high-frequency components of the fast-spike waveform. Analog signals were digitized at $32 \mathrm{kHz}$ using a $1 \mathrm{GHz}$ personal computer equipped with a PCI-MIO-16E-1 board (National Instruments, Austin, TX). Data acquisition software was written in LabView version 5.1.1 (National Instruments). Waveform samples exceeding amplitude thresholds were parsed from the continuous signals, displayed, and stored to disk along with the time stamp (measured at a resolution of $31.25 \mu \mathrm{sec}$ ) and trial information.

At the termination of the experiment, the rat was deeply anesthetized with sodium pentobarbital and perfused transcardially. The cortex was cut tangentially in $60 \mu \mathrm{m}$ frozen serial sections, reacted for cytochrome oxidase $(\mathrm{CO})$, and counterstained with thionine. The thalamus was processed similarly. Using microdrive readings, signs of tissue disruption, and electrolytic lesions, recording sites were localized with respect to individual barrels and, when possible, with respect to individual VPM "barreloids." A cell was classified as a barrel neuron if the recording site was within the CO-rich barrel center in layer IV, which in our hands has consistently been found to correspond to microdrive depth readings of 700-1000 $\mu \mathrm{m}$ (Kyriazi et al., 1996). Control recordings obtained in the thalamic reticular nucleus (TRN) were similarly verified histologically.

Individual large vibrissas of the contralateral mystacial pad were deflected using piezoelectric stimulators (Simons, 1983) controlled by an LSI 11/73 computer (Digital Equipment Corporation, Maynard, MA) or, in later experiments, by the acquisition computer. A stimulator was attached to a whisker $10-12 \mathrm{~mm}$ from the base of the hair, which was deflected using ramp-and-hold movements as described previously (Simons and Carvell, 1989). Whiskers were deflected randomly in each of eight cardinal directions in $45^{\circ}$ increments relative to the horizontal alignment of the whisker rows. The whisker that elicited the strongest responses at successive recording locations was defined as the PW. Twenty blocks of such stimuli were delivered to the PW (160 total stimuli). Ten such blocks were delivered separately to each of the four immediately adjacent whiskers (i.e., those rostral, caudal, dorsal, and ventral to the PW). Unit responses were quantified by measuring the average number of spikes per stimulus occurring during a $20 \mathrm{msec}$ period immediately after the onset of whisker deflection (i.e., ON responses).

Cross-correlation analyses perform best given a relatively large number $(>2000)$ of reference (thalamic) and target (cortical) spikes. To obtain a sufficient number, $1 \mathrm{~mm}$ amplitude $2 \mathrm{sec}$ sinusoidal stimuli of 2 , 4 , or $8 \mathrm{~Hz}$ were applied repeatedly to the PW in a direction for which the ramp-and-hold stimulus reliably elicited spikes from both the thalamic and cortical neurons. These relatively low-velocity sinusoids evoke a substantial number of spikes without strongly synchronizing thalamic neurons (Pinto et al., 2000). Thousands of thalamic and cortical spikes were obtained during 100-300 trials with $3 \mathrm{sec}$ interstimulus intervals.

We recorded data only if spikes were at least three times larger than background noise. Typically, signal-to-noise ratios were on the order of 10:1, and recordings consisted of only a single large-amplitude unit; occasionally, two readily discriminable units were present on a recording channel. Even with the best isolated waveforms, we used spike-sorting techniques to further ensure the quality of single-unit isolation. Spike sorting was performed off-line using MClust version 2.0 (A. David Redish, University of Minnesota, Minneapolis, MN) and our own custom-written programs. This classification method uses amplitude and timing measures of individual waveform maxima and minima as well as the first five principal components, which are sensitive to the overall waveform shape. Our criteria for single units were that (1) for at least one combination of parameters, a cluster of spikes formed a distinct mode, (2) this mode had little or no overlap with any other cluster, and (3) interspike interval histograms revealed few $(<0.1 \%)$ or no spikes occurring within $2 \mathrm{msec}$ of one another.

The presence and strength of thalamocortical connections at monosynaptic latencies were inferred using cross-correlation analysis (Perkel et al., 1967). Firing rates of single units were first checked for stationarity by plotting the total number of spikes per trial. If either the thalamic or cortical cell exhibited any overall trend of increasing/decreasing firing rate, the pair was excluded from the study. For each recorded pair, a "raw" cross-correlogram with $1 \mathrm{msec}$ bins was computed by measuring the time of occurrence of each cortical spike relative to that of each thalamic spike. The following numerical procedure was used to remove stimulus-locked correlation and to determine whether any remaining peak was statistically significant and therefore evidence for a neural connection.

Using Splus 2000 Pro (MathSoft, Cambridge, MA), confidence limits were estimated nonparametrically by a standard bootstrap method (Efron and Tibshirani, 1991; Davison and Hinkley, 1997). Trials were resampled randomly with replacement for each cell, yielding a resampled cross-correlogram, which reflects only stimulus-locked correlation. The difference of the raw and resampled cross-correlograms was found. The process of resampling and calculating differences was iterated 5000 times, and the median of difference values for each time bin was taken as a measure of correlation independent of the stimulus. Confidence limits for this estimate were derived for each bin by finding the 1st and 99th percentile of the distribution of difference values of that bin. If the lower confidence limit was greater than a difference value of 0 , the bin was deemed statistically significant (i.e., one-tailed $p<0.01$ ). For example, in Figure $3 A$, bins 2,3 , and 6 (asterisks) are significantly different from 0 , as indicated by the corresponding points on the lower gray line with values $>0$. A thalamocortical connection was inferred if any of the three bins corresponding to a cortical latency of $1.0-4.0 \mathrm{msec}$ were significant; this corresponds to $p<0.03$ for observing a false positive.

Efficacy of connection was measured as $N_{\mathrm{c}} / N_{\mathrm{pre}}$, where $N_{\mathrm{c}}$ is the sum of the three bins from 1.0 to $4.0 \mathrm{msec}$ in the corrected cross-correlogram and $N_{\text {pre }}$ is the total number of thalamic spikes (Levick et al., 1972). Strength, a less commonly used measure that also takes into account the overall firing rate of the postsynaptic neuron, was measured as follows:

$$
\frac{N_{\mathrm{c}}}{\sqrt{\left(N_{\text {pre }}+N_{\text {post }}\right) / 2}}
$$

where $N_{\text {post }}$ is the total number of cortical spikes (Alonso and Martinez, 1998). The results of all analyses involving efficacy were virtually equivalent when the measure of strength was substituted. Note that neither empirical estimate is intended to measure EPSP magnitude at single synapses. The measures are used only as a means of comparing the relative impact of thalamic contacts, inferred from the cross-correlation analysis, on FSUs versus RSUs.

Tests and SEs of proportions were calculated using a normal approximation to a binomial distribution (Moore and McCabe, 1993). Distributions were compared using the nonparametric Wilcoxon rank-sum (Mann-Whitney) test. The relationship of a continuous variable to a binary one (i.e., connected/unconnected) was tested by logistic regression (logit), which fits a sigmoid to the probability of a positive outcome (Hamilton, 1991).

\section{RESULTS}

\section{Waveform-based classification of cortical cells}

Cortical units were classified as FSUs or RSUs by the time course of a random sample of action potentials recorded for each unit. Two components of the waveform were quantified (Fig. 1A): (1) 
Figure 1. Cortical cell type can be inferred from extracellular waveform shape. $A$, Example waveforms of an RSU and an FSU. Dashed line indicates baseline; 1 and 2 denote initial and secondary phases. $B$, Scatterplot of width measurements of initial and secondary phases. Filled circles, examples shown in A. AHP, Afterhyperpolarization.
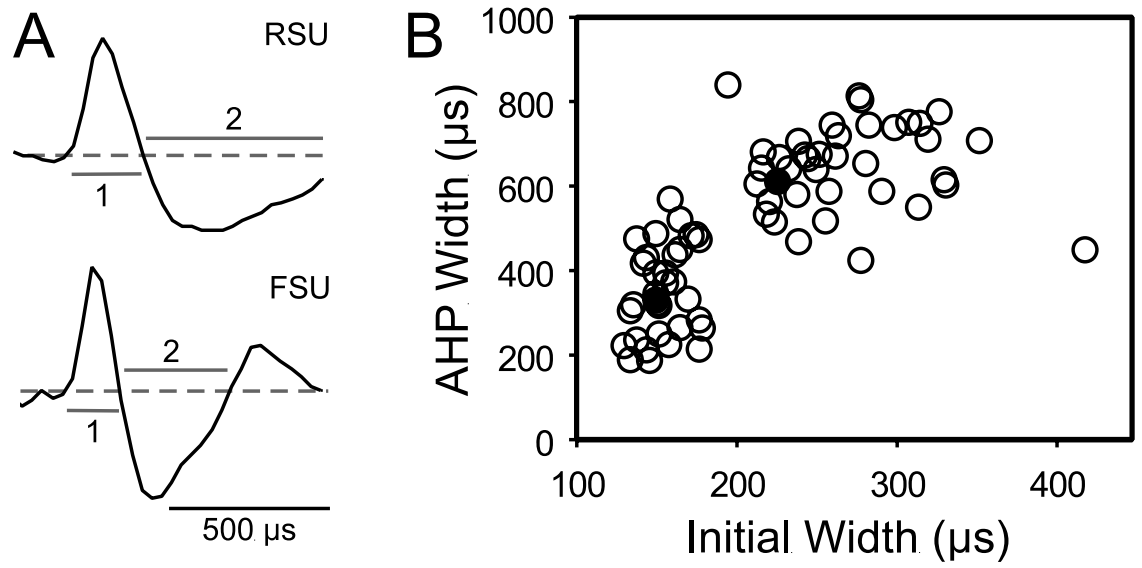

the duration of the initial (negative or positive) wave, measured from its onset to its recrossing of baseline, and (2) the duration of its second phase (corresponding to spike afterhyperpolarization), measured from the end of the first wave to the return to baseline. Their sum is the total spike duration. Figure $1 B$ shows a scatterplot of the mean durations of 71 cells. The cluster with short initial (mean, $155 \mu \mathrm{sec}$; range, 130-179) and secondary (mean, $349 \mu \mathrm{sec}$; range, 185-567) phases was designated as FSUs. All other units were designated as RSUs; these have longer initial (mean, $268 \mu \mathrm{sec}$; range, 195-418) and secondary (mean, 646 $\mu$ sec; range, 422-837) phases. The values for both groups correspond closely to previous qualitative descriptions of extracellularly and intracellularly recorded FSU and RSU waveforms (Simons, 1978; McCormick et al., 1985) and measurements of total extracellular waveform duration (Armstrong-James et al., 1993).

\section{RF properties}

Data were obtained from 95 thalamocortical units (TCUs), 54 RSUs, and 45 FSUs. The response properties of the sampled units were comparable with those described previously (Simons and Carvell, 1989; Armstrong-James et al., 1993; Kyriazi et al., 1994). Evoked firing rates were largest for FSUs, smallest for RSUs, and intermediate for TCUs (mean, 3.2, 1.3, and 1.4 spikes per PW deflection, respectively). RFs were assessed by controlled deflections, in eight cardinal directions, of the PW to which the pair responded and of the four AWs: those immediately caudal, rostral, ventral, and dorsal. Figure 2 shows measures of directional tuning and RF size. Directional sensitivity, quantified as the ratio of the response to the maximally effective deflection angle to the average response over all eight angles, was smallest for FSUs, which are the least tuned, largest for RSUs and intermediate for TCUs (means, 1.6, 2.0, and 1.8, respectively) (Fig. 2A,C,E).

Similarly, FSUs have the largest RFs (Fig. $2 B, D, F$ ). RF size was measured as the number of whiskers (PW and up to four AWs) evoking a response statistically greater than spontaneous activity (Simons and Carvell, 1989). Of the 32 FSUs for which all five whiskers were tested, only two had single-whisker RFs; of the 30 multi-whisker FSUs, the average number of responsive AWs was 2.3, evoking responses that were on average $43 \%$ as large as their PWs. Complete RF maps were obtained for 31 regular-spike barrel neurons. Fifteen RSUs had single-whisker RFs; of the 16 multi-whisker RSUs, the average number of responsive AWs was 2.0, evoking responses that were on average $27 \%$ as large as their PWs.
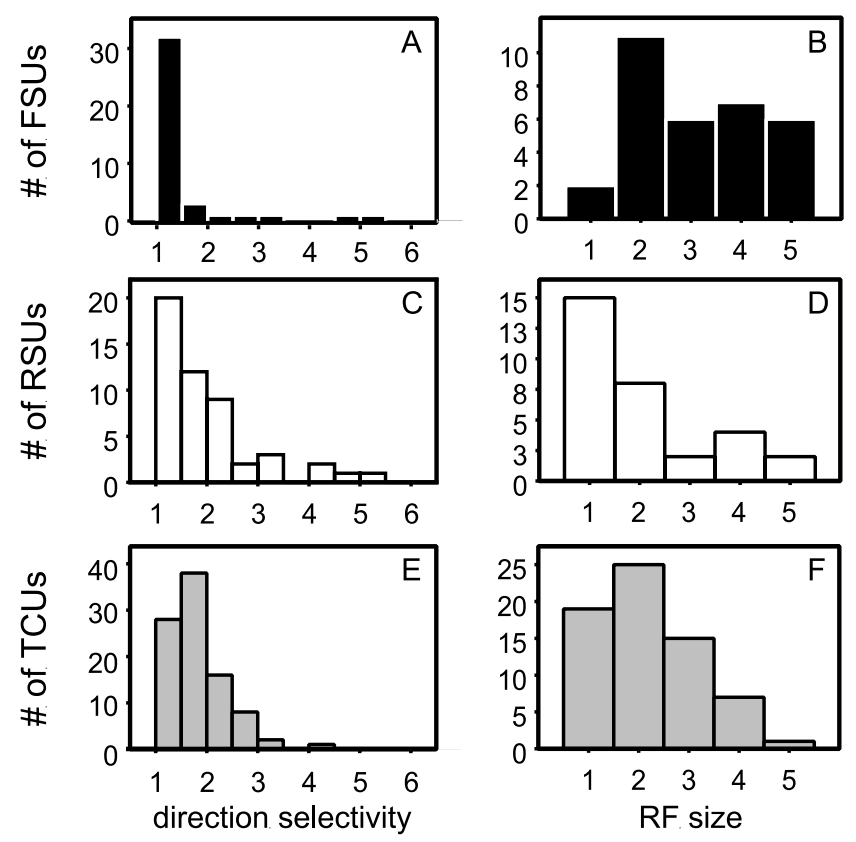

Figure 2. RF properties of FSUs $(A, B)$, RSUs $(C, D)$, and TCUs $(E, F)$. $A, C, E$, Histograms of the direction selectivity index (response to whisker deflection at angle evoking the maximum number of spikes per average response over all eight angles). $B, D, F$, Histograms of number of whiskers evoking a response significantly greater than spontaneous activity.

\section{Thalamocortical connectivity of FSUs and RSUs}

Cross-correlation analysis was used to infer connections between pairs of simultaneously recorded thalamocortical and cortical layer IV neurons. Similar to other studies (Reid and Alonso, 1995; Swadlow, 1995; Miller et al., 2001), our criterion for a monosynaptic thalamocortical connection was a statistically significant, increased probability of cortical spiking $1.0-4.0 \mathrm{msec}$ after thalamic cell discharge. Note that subthreshold inputs can be detected by this approach, because postsynaptic EPSPs influence the probability of cortical discharge. Figure 3 shows crosscorrelograms providing evidence of connections for a TCU-FSU pair (Fig. 3A) and a TCU-RSU pair (Fig. 3B). The peaks at the $+2 \mathrm{msec}$ bins are significant, and the elevated correlation falls off quickly. Later peaks, such as the $+6 \mathrm{msec}$ bin in Figure $3 A$, may reflect the shape of the underlying EPSP, bursting of the postsynaptic cell, and/or polysynaptic connections between the paired 

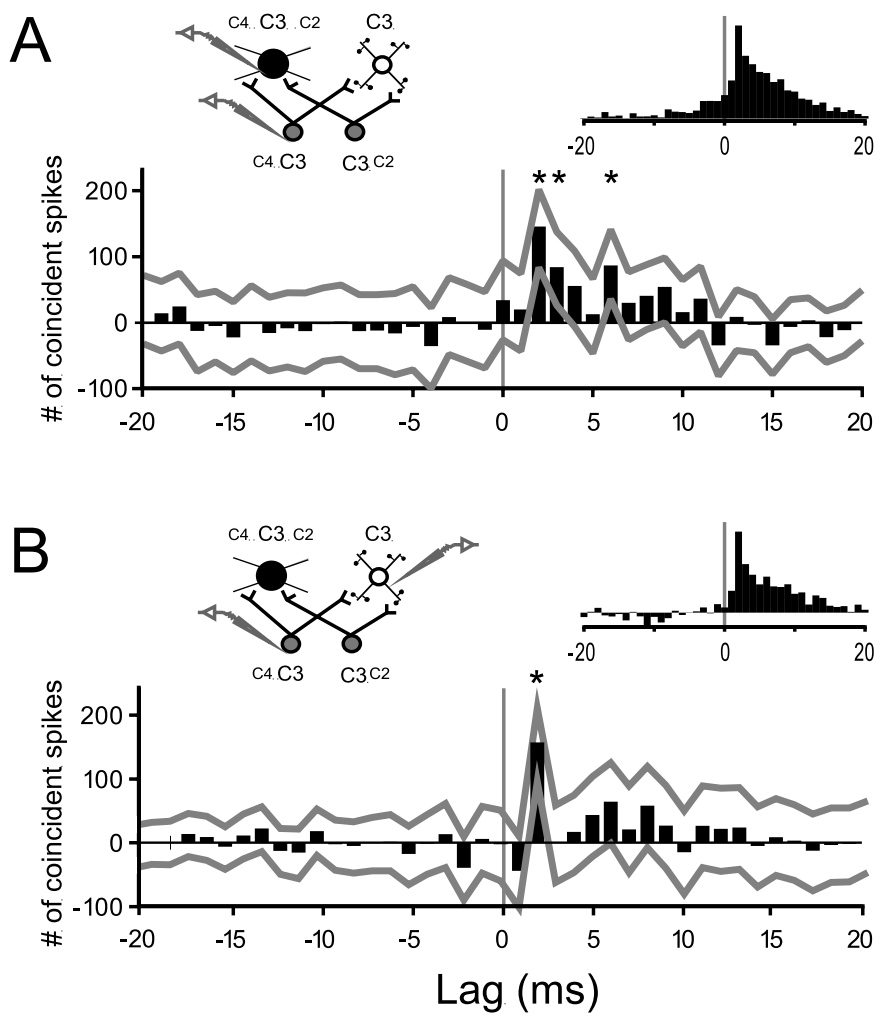

Figure 3. Representative and average cross-correlograms of thalamocortical pairs. $A$, Representative stimulus-corrected cross-correlogram for a connected TCU-FSU pair. $x$-axis, Milliseconds by which cortical spikes follow (positive lags) or precede (negative lags) reference thalamic spikes. $y$-axis, Number of coincident spikes relative to baseline (see Materials and Methods). Gray lines indicate $99 \%$ confidence limits for individual bins. In this example, the $+2,+3$, and +6 bins (asterisks) are significantly $>0$ (baseline). $N_{\text {pre }}=2427$ spikes; $N_{\text {post }}=9412$ spikes. Left inset, Experimental design and hypothetical circuit showing multi-whisker thalamic neurons (gray), an inhibitory cortical neuron with a larger RF (black), and an excitatory neuron with a smaller RF (white). Right inset, Average of peak-normalized cross-correlograms of all putatively connected TCUFSU pairs. $B$, Same as $A$, but for a connected TCU-RSU pair $\left(N_{\text {pre }}=\right.$ 11,090 spikes; $N_{\text {post }}=8036$ spikes) and the average of all TCU-RSU pairs.

neurons. The small bin values at almost all other lag times are not statistically different from 0 (baseline correlation). The mean lag time of significant peak maxima was $+1.6 \pm 0.098 \mathrm{msec}$, consistent with known delays between thalamic and cortical spiking (Kyriazi et al., 1994). To ensure that significant early peaks were not attributable to bursting, correlograms were also computed after deleting thalamic and cortical spikes with short $(<10 \mathrm{msec})$ interstimulus intervals. The inference of monosynaptic connectivity was in no case affected by the removal of burst spikes. All significant cross-correlogram peaks occurred at early positive lag times and did not cross the 0 lag time, as exemplified by Figure 3 .

Averaged normalized cross-correlograms were constructed separately for all connected TCU-RSU and TCU-FSU pairs and were qualitatively similar to each other (Fig. 3, right insets). The peak in the average of connected TCU-FSU data does slightly cross 0 , because FSUs are more likely to burst, thereby inducing small, although statistically nonsignificant, elevations at lag times preceding the monosynaptic window (e.g., the -1 to -10 bins). Within RSU and FSU groups, waveform parameters were not related to the likelihood of connection $(p>0.65)$.

Connections were observed only when thalamic and cortical
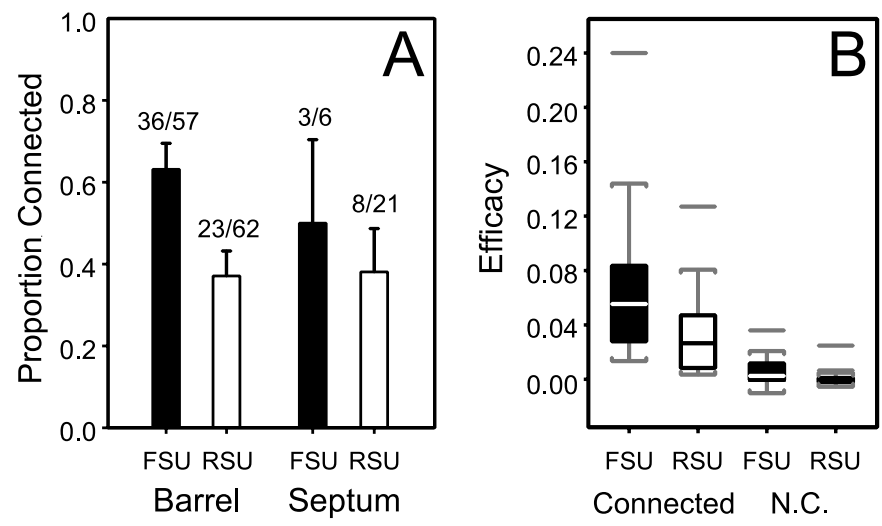

Figure 4. RSUs receive both less numerous and weaker thalamocortical connections than do FSUs. $A$, Summary of the proportions of connected pairs observed for each anatomical category studied (see Results). Error bars indicate SEs of the proportion. $B$, Standard box plots of the efficacy of pairs of thalamic and barrel FSUs and RSUs found to be connected and not connected (N.C.). Boxes indicate 25-75 percentile ranges of the distributions; lines through boxes indicate medians; vertical lines indicate tails of distributions; isolated horizontal lines indicates outliers.

neurons were topographically aligned, being maximally responsive to the same PW. Figure $4 A$ shows data for 166 pairs of thalamic and cortical neurons. Of 146 such pairs, recordings were obtained from a topographically aligned cortical barrel $(n=119)$ or from the immediately adjacent "septum" $(n=27)$, the relatively cell-sparse region separating barrels. Seventy pairs $(48 \%)$ were connected, with statistically equivalent proportions for barrel and septal neurons $(p=0.41)$. These latter findings are consistent with septal neurons with dendrites that extend into nearby barrel centers (Simons and Woolsey, 1984; Brumberg et al., 1999). Because of the small sample of connected septal neurons, all remaining analyses apply only to units recorded within barrels.

Several additional analyses provide more controls for artifactual connections. None of the 13 cortical neurons recorded in neighboring barrels, unaligned with the recorded thalamic neuron, exhibited significant peaks. These findings are consistent with the nearly one-to-one anatomical relationship between thalamic barreloids and cortical barrels (Bernardo and Woolsey, 1987; Agmon et al., 1995; Land et al., 1995). The mean spontaneous and stimulus-evoked firing rates of thalamic neurons were equivalent for pairs found to have a connection and those that were not $(p>0.35)$. Simultaneous recordings were also obtained from FSUs and cells in the topographically aligned region of the TRN. TRN neurons, like cells in barreloids, have high spontaneous and stimulus-evoked firing rates (Hartings et al., 2000); however, unlike those in the barreloids, they do not project to cortical layer IV. If the methods were prone to false positives, one would expect at least one of the TRN-FSU pairs to exhibit significant peaks. Of the seven pairs studied, none showed evidence of connections. Finally, as noted in Materials and Methods, we explicitly used low-velocity whisker deflections that are known to evoke relatively asynchronous firing patterns among thalamic neurons, suggesting that inferences about connectivity were specific to the recorded TCU, not another (unrecorded) thalamic cell whose firing was tightly synchronized with it by the stimulus. Similarly, gap junction coupling between cortical FSUs could contribute to false positives, but our use of weakly synchronizing whisker stimuli and the strong attenuation of EPSPs $(\sim 15$-fold) and spikes ( $\sim 100$-fold) at these electrical contacts (Gibson et al., 
TCU-RSU pairs
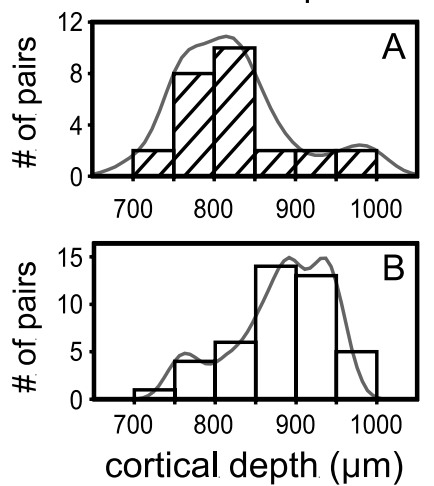

TCU-FSU pairs
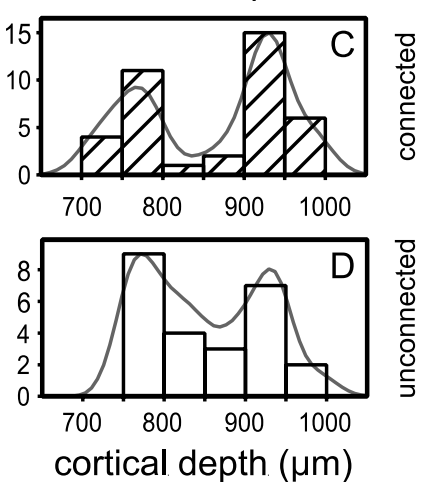

Figure 5. Connected TCU-RSU pairs tend to involve RSUs located superficially in layer IV. Shown are histograms of microdrive depth readings for connected $(A, C)$ and unconnected $(B, D)$ pairs involving RSUs $(A, B)$ and FSUs $(C, D)$.

1999) is likely to minimize the confounding influence of the nonchemical transmission of synchronous spikes among FSUs.

FSUs are nearly twice as likely as RSUs to receive detectable thalamocortical inputs (Fig. $4 A$ ). Of the 57 somatotopically aligned thalamocortical pairs involving barrel FSUs, $63 \%$ were coupled, whereas only $37 \%$ of the 62 pairs involving barrel RSUs were coupled. This difference in the probability of connection is highly significant $(p=0.002)$. This result strongly suggests that an individual barrel FSU is contacted by more somatotopically aligned thalamic neurons than is an RSU.

Using a measure of connection efficacy, we tested the hypothesis that in connected pairs, an action potential arriving along a thalamic axon was more likely to be followed by a spike in FSUs than in RSUs. The most efficacious connections were observed for FSUs (ranges: RSU, 0.0034-0.13; FSU, 0.013-0.24) (Fig. 4B). On average, connected TCU-FSU pairs had significantly higher connection efficacy than connected TCU-RSU pairs (means, 0.063 and 0.034 , respectively; $p=0.0046$ ). As expected, the efficacies computed for unconnected pairs did not differ significantly from 0 . Together, our results suggest that individual excitatory neurons in layer IV receive less convergent, weaker thalamic inputs than do inhibitory cells.

Figure 5 shows the depth distributions, taken directly from microdrive readings, of the recorded barrel neurons. Although RSUs were encountered uniformly throughout, connected TCURSU pairs were found predominantly more superficially ( $p=$ 0.0002 ) (Fig. 5A,B); interestingly, spiny stellate cells with apical processes are most abundant at the layer III-IV boundary (Simons and Woolsey, 1984). FSUs, however, were more likely to be encountered superficially and deep. However, there was no significant depth-related difference in the probability of finding a TCU-FSU connection $(p=0.72$ ) (Fig. 5C,D). The bimodal depth distribution of sampled FSUs closely resembles that of Golgi-impregnated barrel neurons with smooth dendrites, which are found more often at the layer III-IV and IV-V boundaries (Simons and Woolsey, 1984). Together, the RSU and FSU results provide additional confirmation of the accuracy of the depth readings and of the classification of putative excitatory and inhibitory neurons on the basis of spike waveforms. They also suggest that excitatory barrel neurons located in upper layer IV may be functionally different from those residing more deeply.

We examined whether FSUs and RSUs receive input from similar populations of thalamic neurons. TCUs connected to

Connected TCU-FSU Pairs

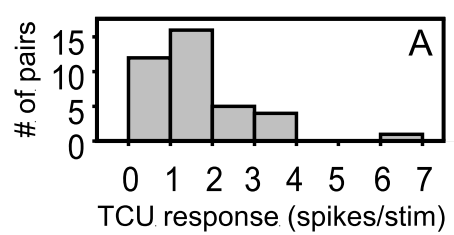

Connected TCU-RSU Pairs
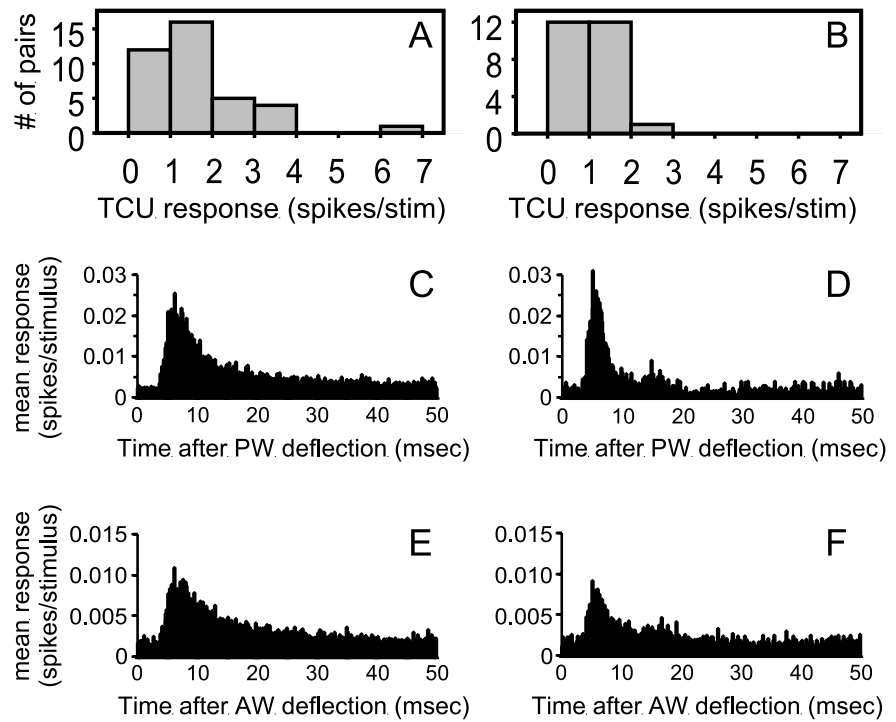

Figure 6. RSUs but not FSUs receive inputs exclusively from thalamic neurons with low evoked firing rates. $A, B$, Distributions of responses to PW deflections for thalamic neurons found to be connected to FSUs $(A)$ and RSUs $(B) . C-F$, Average PSTHs of thalamic responses to PW $(C, D)$ and AW $(E, F)$ deflections for FSUs $(C, E)$ and RSUs $(D, F)$. Bin width is $100 \mu \mathrm{sec}$.

FSUs did not differ from those connected to RSUs in terms of spontaneous activity, direction selectivity, or RF size $(p>0.25)$. Unexpectedly, FSUs receive inputs from thalamic neurons that, on average, have stronger responses to $\mathrm{PW}$ deflection $(p=0.02)$. As shown in Figure $6 A, B$, this difference is attributable to RSUs receiving connections exclusively from TCUs with less robust responses, whereas FSUs are contacted by both weakly and strongly responsive TCUs. Average peristimulus time histograms (PSTHs) show that TCUs connected to FSUs have longer-lasting responses to $\mathrm{PW}$ deflections (Fig. 6C,D); they also discharge more spikes during the latter $100 \mathrm{msec}$ of the $200 \mathrm{msec}$ plateau phase of whisker deflection $(p=0.049)$. The response profiles of the TCUs contacting FSUs more closely resemble previous samples of the entire thalamic population (Simons and Carvell, 1989; Pinto et al., 2000) than does the more restricted set of TCUs that contacts RSUs. Interestingly, the average firing rates of the two thalamic populations are similar during the first $5 \mathrm{msec}$ of the response $(p=0.66)$, the period of maximal sensitivity of barrel circuitry to thalamic input (Pinto et al., 2000). Similar, although somewhat less pronounced, differences exist for AW responses (Fig. 6E,F).

\section{Connectivity and RF properties}

FSUs have larger, less directionally tuned RFs than either RSUs or TCUs. The large RFs of inhibitory barrel neurons could be explained by their receiving inputs primarily from multi-whisker TCUs, and the smaller RFs of excitatory cells could reflect inputs from primarily single-whisker TCUs. Therefore, we analyzed presynaptic and postsynaptic RF sizes for connected and unconnected pairs of TCUs and FSUs. TCUs with small or large RFs were equally likely to be connected to FSUs (Fig. 7A, filled triangles) (logit, $p=0.92$ ), and TCU RF sizes were equivalent for connected and unconnected pairs $(p=0.86)$. Similarly, RSUs were contacted with equal probability by TCUs of all RF sizes (Fig. $7 A$, open triangles; logit, $p=0.32$ ). Thus, the RF size of a 

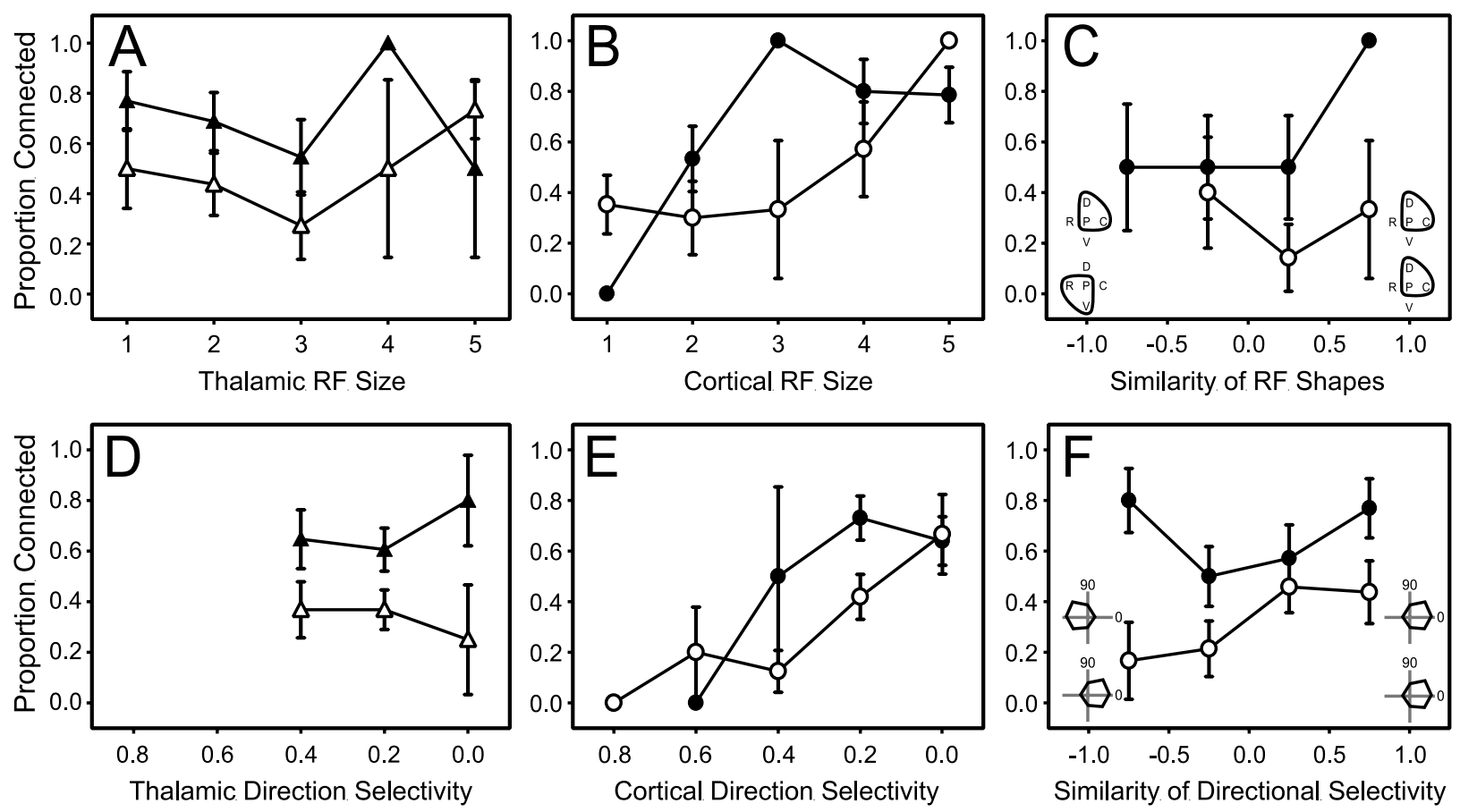

Figure 7. RF size and direction selectivity of cortical layer IV neurons reflect the amount of nonspecific convergence of thalamocortical input. $A-F$, open symbols, TCU-RSU pairs; filled symbols, TCU-FSU pairs. Error bars indicate SEs of the proportion. Connected proportion of pairs as a function of $A$, the RF size (number of responsive whiskers) of the thalamic neuron. $B$, The RF size of the cortical neuron. $C$, The similarity of thalamic and cortical RF shapes with insets schematically depicting examples of perfectly nonmatching (left) and matching (right) thalamic and cortical RF shapes. $P$, Principal whisker; $C, D, R$, and $V$, caudal, dorsal, rostral, and ventral AWs, respectively; lines, more effective whiskers. $D$, The directional tuning (in log units) of the response of the thalamic neuron to PW deflections. $E$, The directional tuning (in log units) of the responses of the cortical neuron to PW deflections. $F$, The similarity (see Results) of the responses of a thalamocortical pair to PW deflections in each of eight directions, with insets schematically depicting examples of anticorrelated (left) and correlated (right) response profiles. O, Caudal deflections; 90, dorsal deflections. D, E, Although moderately directionally selective, TCUs have a more limited range of tuning than RSUs. Likewise, tuned FSUs exist, but not in the extreme. Consequently, some datapoints do not exist for TCUs and FSUs.

given thalamic neuron does not determine the likelihood of its forming a connection with a barrel neuron.

Figure $7 B$ shows the probability of finding a thalamocortical connection as a function of the RF size of the cortical neuron. For FSUs, cortical RF size influences connection probability, such that FSUs with larger RFs are more likely to receive TCU connections (Fig. 7B, filled circles; logit, $p=0.035$ ). Connections were observed for less than half of TCU-FSU pairs with relatively small FSU RFs [i.e., limited to the PW $(n=1)$ or the PW and one AW $(n=15)]$, whereas the probability of connection was $\sim 85 \%$ for those responding to two or more AWs $(n=31)$. This relationship of FSU RF size and observed TCU connectivity is not attributable to differences in postsynaptic cell excitability. The spontaneous and evoked firing rates of FSUs of RF size 1 (i.e., PW only) and size 2 (i.e., PW and one AW) were similar to those of sizes 3-5 ( $p=0.61$ and 0.29 , for spontaneous and evoked activities, respectively). The likelihood of finding TCU-FSU connections was higher for pairs of cells with similarly shaped RF surrounds, measured by the relative contributions of each of the four AWs (Fig. 7C). For RSUs, the relationship of cortical RF size and the probability of observing a connection was significant only at a trend level (Fig. 7C, open circles; logit, $p=0.081$ ); no significant relationship was observed between connectivity and surround organization. These negative results may be attributable to the rarity of RSUs with large (more TCU-like) RFs. Together, the findings show that cortical RF size is proportional to the amount of convergent input from topographically aligned TCUs with variable RF size. The difference in RF size between FSUs
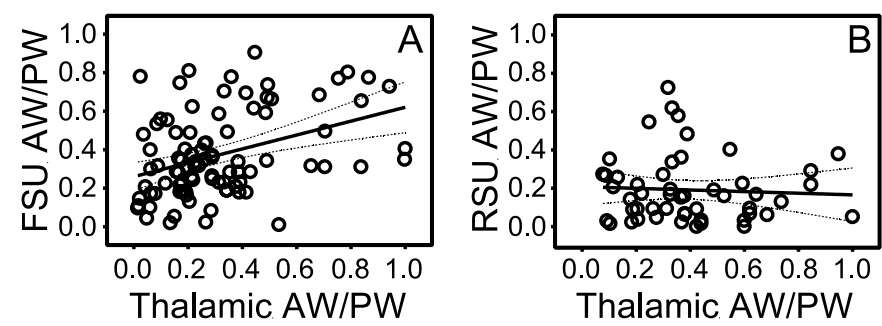

Figure 8. RFs of inhibitory but not excitatory barrel neurons have shapes similar to those of their thalamic inputs. A, Relationship of AW/PW response ratios for connected pairs of TCUs and FSUs. The AW/PW ratio is defined as the mean response of a neuron to the deflection of an AW over the mean response to deflection of its PW. $B$, Same as $A$, but for connected pairs of TCUs and RSUs. $A$, $B$, solid line, Fitted linear regression; dottedlines, $95 \%$ confidence limits. Note that both FSUs and RSUs are contacted by TCUs with focused (small ratio) and unfocused (large ratio) RFs.

and RSUs is not attributable to their receiving inputs exclusively from thalamic neurons with large and small RFs, respectively.

As noted above, all connected thalamic and cortical neurons had the same PW or RF center. We also analyzed RF organization by comparing the contribution of corresponding AWs in connected pairs. Each response of a TCU to an AW deflection was normalized to its $\mathrm{PW}$ response; this $\mathrm{AW} / \mathrm{PW}$ ratio was linearly regressed against the corresponding ratio for the connected cortical neuron. As shown in the scatterplots of Figure 8, distinctly different relationships were observed for FSUs and RSUs. 
Figure 9. Thalamic neurons with spatial response biases are more likely to contact FSUs. $A$, Histograms of dorsoventral response biases of TCUs classified as connected (filled columns) and unconnected (open columns). Cells responding only to dorsal AW deflections have a bias of -1.0 ; those responding only to ventral AW deflections have a bias of +1.0 . Biases of 0 indicate no difference between dorsal and ventral AW deflections. $B$, Same as $A$, but for the rostrocaudal axis.
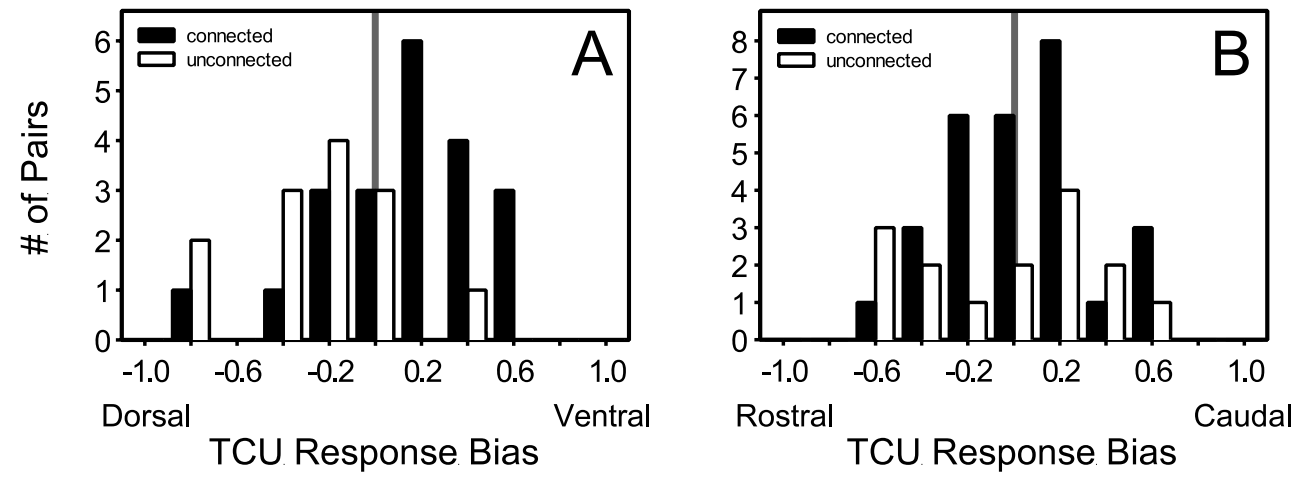

In a connected TCU-FSU pair, the specific AW responses of the thalamic unit are expressed in the RF of the FSU $\left(r^{2}=0.15 ; p=\right.$ $0.0002)$. In contrast, the shape of the RF of an RSU is independent of the thalamic RF $\left(r^{2}=0.003 ; p=0.70\right)$, except that both have the same PW. Note, for example, that the AW/PW ratios of RSUs remain small despite inputs from TCUs with relatively unfocused RFs (e.g., ratios of $>0.5$ ). Thus, FSUs reflect the RF combination of their TCU inputs (PW and AWs), whereas RSUs reflect their commonality (i.e., PW only).

Analogous relationships exist for the direction selectivity of PW responses. Directionally selective TCUs were as likely to contact cortical neurons (RSUs or FSUs) as were weakly selective ones (Fig. 7D; FSUs: filled triangles, logit, $p=0.89$; RSUs: open triangles, logit, $p=0.81)$. Moreover, cortical neurons that responded strongly to multiple deflection angles were more likely to receive a thalamic connection than cells that responded strongly to only a few directions (Fig. 7E; FSUs: filled circles, logit, $p=$ 0.029; RSUs: open circles, logit, $p=0.012$ ). Thus, as in the case of RF size (Figs. 7A,B), directional tuning is related to the amount of convergent input from TCUs with variable degrees of directional selectivity, with broadly tuned cortical cells receiving the most convergence. Nevertheless, the directional preferences of cortical neurons may reflect their thalamocortical inputs. For each recorded pair, we compared the similarity of their directional tuning by computing a correlation coefficient based on the corresponding responses of the two cells to each of the eight deflection angles; a value of 1.0 indicates identical directional tuning; a value of -1.0 indicates complete dissimilarity (Fig. $7 F$, insets). For the 62 TCU-RSU pairs, the probability of finding a connection was proportional to the similarity of the tuning curves (Fig. $7 F$, open circles, logit, $p=0.03$ ). No relationship was observed for the 57 TCU-FSU pairs (Fig. $7 F$, filled circles, logit, $p=0.20$ ), perhaps attributable in part to the rarity of FSUs with tuned (more TCU-like) directional preferences. The influence of RF shape similarity on TCU-FSU connections (Fig. 7C) and the influence of directional tuning similarity on TCU-RSU connections (Fig. $7 F$ ) may be analogous to recent observations in the visual system that RF properties tend to be matched in connected pairs (Alonso et al., 2001).

\section{Connectivity and TCU receptive-field asymmetry}

The TCUs, RSUs, and FSUs sampled all displayed, on average, stronger responses to caudal than rostral AWs and even more pronounced biases toward ventral versus dorsal AWs. Previous studies showed that the response of a cortical neuron to PW deflection is substantially suppressed if it is preceded by the conditioning stimulation of an AW; effects are anisotropic, in that caudal AWs are more suppressive than rostral, and ventral more than dorsal (Brumberg et al., 1996). Therefore, we examined whether TCUs with rostrocaudal or dorsoventral biases were more likely to contact FSUs and RSUs. For each TCU studied, a spatial response bias was calculated as follows: bias $=$ $\left(O N_{\text {ventral }}-O N_{\text {dorsal }}\right) /\left(O N_{\text {ventral }}+O N_{\text {dorsal }}\right)$, where $O N_{i}$ is the ON response of whisker $i$ averaged over all eight deflection angles. An analogous formula was used for the rostrocaudal axis. Figure $9 A$ shows that connected TCU-FSU pairs were likely to have a ventral response bias within their TCU RFs, whereas unconnected pairs tended to be dorsally biased (one-tailed $p=$ 0.0017). No significant difference was found between connected and unconnected TCUs for the rostrocaudal axis (Fig. 9B) (onetailed $p=0.32$ ). In this study, as in others (see Discussion), the spatial gradient was stronger in the dorsoventral dimension than in the rostrocaudal dimension; the sample size here may simply be too small to detect the potentially more subtle difference along the rostrocaudal axis. Preferential connectivity was not observed for RSUs (data not shown). Thus, the excitatory spatial gradients of FSUs may be enhanced by preferential connectivity and, via feedforward inhibition, subsequently manifested in the inhibitory subfields of excitatory neurons.

\section{Connection efficacy}

We examined whether connection efficacy, like connection probability, was related to RF properties. For all connected pairs, efficacy was linearly regressed separately for TCU-RSU and TCU-FSU pairs against RF size, PW directional tuning, spontaneous activity, and mean evoked responses of the thalamic and cortical cells. Also tested were relationships of efficacy and similarity of presynaptic and postsynaptic RFs in terms of size, $\mathrm{AW} / \mathrm{PW}$ ratios, or PW directional tuning. None of these relationships were even mildly significant except for RSU spontaneous $\left(r^{2}=0.68 ; p=0.0001\right)$ and evoked firing rates $\left(r^{2}=0.2 ; p=\right.$ $0.039)$; higher postsynaptic firing rates are expected given stronger connections. Thus, as reported by others (Reid and Alonso, 1995; Miller et al., 2001), connection efficacy, unlike connection probability, does not relate to any obvious RF property in this circuit (but cf Alonso et al., 2001).

\section{DISCUSSION}

Within the barrel, fast-spike, presumably inhibitory, cells receive more detectable connections than regular-spike, presumably excitatory, neurons. The high proportion (63\%) of connected FSUs is comparable with the $65 \%$ thalamocortical connectivity of suspected inhibitory neurons (SINs) in rabbit barrel cortex (Swadlow, 1995; Swadlow and Gusev, 2002). SINs, FSUs with an average waveform duration of $\sim 0.36 \mathrm{msec}$, are defined by their high-frequency responses (more than or equal to three spikes at 
$>600 \mathrm{~Hz}$ ) to electrical thalamic stimulation; these may correspond to our most strongly driven FSUs. Our FSU sample includes both units that would be classified as SINs as well as those whose response characteristics would not meet the SIN criteria. The high probability of encountering connections onto FSUs, including SINs, reflects the high convergence of thalamocortical neurons. In contrast, only $37 \%$ of thalamocortical pairs involving RSUs exhibited connections. Because a connection can be detected by cross correlation only if its influence is large enough to produce a correlogram peak that exceeds noise, which is often substantial in these analyses, RSUs may receive connections undetected here. It remains to be determined whether the lower TCU-RSU connection probability is attributable to weak connections and/or to sparse anatomical connectivity. Studies in cat visual cortex (Tanaka, 1985; Reid and Alonso, 1995) and rat somatosensory cortex (Johnson and Alloway, 1996) reported overall thalamocortical connection probabilities $(\sim 30 \%)$, similar to our estimate for barrel RSUs. Cortical units were not classified by criteria that might have distinguished excitatory from inhibitory cells. Because electrodes are biased toward various cell types, those studies may have sampled predominantly excitatory neurons.

Thalamocortical synapses onto smooth and spiny barrel cells were first demonstrated by electron microscopy (White, 1978; White et al., 1984; Keller and White, 1987), and their potencies were explored electrophysiologically in vitro (Agmon and Connors, 1991, 1992; Porter et al., 2001). Given a density of neurons of $\sim 112,000 / \mathrm{mm}^{3}$ in adult rat layer IV (Keller and Carlson, 1999) and the assumption of a volume of $0.035 \mathrm{~mm}^{3}$ (Welker and Woolsey, 1974), a barrel contains $\sim 4000$ neurons. Because no more than $10 \%$ are inhibitory (Beaulieu, 1993) and not all inhibitory cells are FSUs (Kawaguchi and Kubota, 1993; Gibson et al., 1999), our findings suggest that each TCU influences $\sim 1300$ RSUs $(3600 \times 0.37)$ and $\sim 250$ FSUs $(400 \times 0.63)$. Assuming $\sim 250$ neurons per barreloid (Land et al., 1995), a fast-spike neuron is likely to be influenced, on average, by $\sim 150$ thalamocortical neurons $(250 \times 0.63)$, but a regular-spike cell by only $\sim 90$ thalamocortical neurons $(250 \times 0.37)$; although thalamocortical synapses onto somata and proximal dendritic segments of inhibitory neurons have been examined (White et al., 1984; Keller and White, 1987), the total number is unknown. Higher FSU connection probability could result from greater numbers of direct thalamocortical connections and/or via indirect gap-junctionmediated electrical coupling among FSUs (Gibson et al., 1999). Disregarding the possible contribution of gap junctions on the grounds of their high signal attenuation and our use of weakly synchronizing stimuli (see Results), the different degrees of thalamocortical convergence onto FSUs and RSUs observed here may reflect the relative sizes of their dendritic arbors. Indeed, smooth cells have larger dendritic trees than spiny cells (Woolsey et al., 1975; Simons and Woolsey, 1984).

Fast-spike barrel neurons in vivo receive more efficacious thalamocortical connections than do regular-spike neurons. This finding mirrors the observation in vitro that microstimulation of thalamocortical axons evokes EPSPs in fast-spike cells with amplitudes approximately twofold larger than in regular-spike cells (Gibson et al., 1999); this may reflect, in part, the presence of thalamocortical synapses on the somata of inhibitory (Keller and White, 1987) but not excitatory (Benshalom and White, 1986) neurons. Furthermore, the overall low efficacy reported here and previously (Swadlow, 1995; Johnson and Alloway, 1996) indicates that a single thalamocortical spike has approximately a 1 in 20 chance of being followed directly by a cortical spike. Thus, in vivo, as in vitro (Porter et al., 2001), individual thalamocortical spikes produce primarily subthreshold EPSPs.

\section{Response properties of barrel FSUs and RSUs}

As in previous studies (Simons and Carvell, 1989; ArmstrongJames et al., 1993; Kyriazi et al., 1994), FSUs and RSUs exhibited different RF properties. FSUs have higher spontaneous and evoked firing rates, less directional selectivity, and larger RFs. The present findings implicate several feedforward mechanisms that contribute to these differences. FSUs are twice as likely as RSUs to receive a detectable thalamocortical connection, and such connections onto FSUs are twice as strong as those onto RSUs. Also, FSUs were found to be contacted by an additional population of strongly driven TCUs that do not appear to contact RSUs.

Differences in RSU and FSU RF properties are consistent with their relative innervations and connection strengths. Large RF size is one consequence of high, nonspecific convergence from a thalamic population with small and large RFs. Indeed, we found that FSU RFs were at least as large as, and often larger than, the RFs of the coupled thalamic neurons, and that FSUs received inputs from thalamic neurons of various RF sizes. Also, the likelihood of observing a thalamic input was proportional to the FSU RF size and was inversely related to FSU directional tuning. These findings and those from a recent cross-correlation study of directional sensitivity in another species (Swadlow and Gusev, 2002) support the proposal (Simons and Carvell, 1989; Swadlow, 1995) that the weak directional selectivity and large RFs of inhibitory barrel cells reflect a combination of their efficacious, convergent thalamic inputs. The characteristic high activity levels of FSUs are likely generated by intrinsic neuronal properties (e.g., depolarized resting membrane potential, low spike thresholds, and/or short absolute refractory periods) (McCormick et al., 1985; Kawaguchi and Kubota, 1993; Rudy et al., 1999) as well as their receipt of inputs from thalamic neurons with high activity levels.

RSUs were less likely than FSUs to receive detectable thalamic connections; these connections were, on average, weaker. Like FSUs, RSUs received inputs from thalamic neurons with a wide range of RF sizes (Figs. 7, 8), but unlike FSUs, they were less likely to express AW-evoked activity in their spike discharge. Limited thalamic convergence and relatively weak connections onto excitatory cells could produce, along with intrinsic membrane properties and fast local inhibition (Simons and Carvell, 1989; Porter et al., 2001), smaller RFs in RSUs, greater directional tuning, and lower spontaneous and stimulus-evoked activities. We found that RSUs receive inputs exclusively from thalamic neurons with comparatively weak responses; this may also contribute to their low stimulus-evoked activities. Similarly, the greater directional tuning of RSUs may also reflect the more limited range of directional preferences among their respective TCU inputs.

The stimulus-evoked firing rates of individual TCUs appear to be well matched to the overall firing rates of their respective cortical targets. Only FSUs are contacted by the most strongly driven TCUs. This may be a consequence of developmentally regulated spike timing-dependent plasticity (Bi and Poo, 1998), which strengthens synapses between cells with correlated firing. FSUs are intrinsically capable of spike rates comparable with or exceeding those of strongly driven TCUs. Therefore, highly active thalamic neurons may be more likely to maintain synapses 
with FSUs than with RSUs. This mechanism may also account for the greater convergence and efficacy of thalamocortical connections onto inhibitory neurons. In this regard, the more robust responses and larger RFs of barrel RSUs in neonatally whiskertrimmed animals (Simons and Land, 1987) may be a consequence of decreased thalamic activity. Lower activity would not exceed the firing capabilities of excitatory neurons, permitting them to maintain strong contacts with greater numbers of thalamic neurons, including those that will become the most active on whisker regrowth.

\section{Feedforward inhibition within local cortical circuits}

Barrel RSUs are almost uniformly subject to the strong AWevoked inhibition (Simons and Carvell, 1989; McCasland et al., 1991; Brumberg et al., 1996) that persists after the ablation of adjacent barrels (Goldreich et al., 1999). Such prominent inhibitory subfields are less frequent in thalamic relay neurons. The present findings suggest that the convergence of strong inputs from neurons in the somatotopically aligned barreloid constructs large, multi-whisker RFs in inhibitory barrel neurons. These, in turn, generate inhibitory subfields in nearby excitatory cells. The present findings similarly provide a feedforward mechanism for subtle spatial gradients of inhibitory subfields. Namely, the tendency for caudally/ventrally biased TCUs to contact FSUs more frequently than rostrally/dorsally biased ones do would generate the known anisotropic inhibitory influences of AWs.

We conclude that effective thalamocortical convergence is substantially greater for fast-spike, inhibitory barrel neurons than for regular-spike, presumed excitatory cells; that individual connections onto inhibitory cells are stronger than those onto excitatory ones; and that evoked firing rates are, on average, stronger for thalamic neurons contacting inhibitory cells. The end result is a greater net thalamic drive to inhibitory barrel circuitry, a crucial component of RF synthesis and response tuning of excitatory cells at the earliest stage of cortical processing. Such strong feedforward inhibition renders barrel circuitry particularly sensitive to the initial firing synchrony of thalamic neurons (Kyriazi and Simons, 1993; Pinto et al., 1996), produced for example by high-velocity deflections of the PW (Pinto et al., 2000, 2002).

\section{REFERENCES}

Agmon A, Connors BW (1991) Thalamocortical responses of mouse somatosensory (barrel) cortex in vitro. Neuroscience 41:365-379.

Agmon A, Connors BW (1992) Correlation between intrinsic firing patterns and thalamocortical synaptic responses of neurons in mouse barrel cortex. J Neurosci 12:319-329.

Agmon A, Yang LT, Jones EG, O'Dowd DK (1995) Topological precision in the thalamic projection to neonatal mouse barrel cortex. J Neurosci 15:549-561.

Alonso J-M, Martinez LM (1998) Functional connectivity between simple cells and complex cells in cat striate cortex. Nat Neurosci 1:395-403.

Alonso J-M, Usrey WM, Reid RC (2001) Rules of connectivity between geniculate cells and simple cells in cat primary visual cortex. J Neurosci 21:4002-4015.

Angulo MC, Rossier J, Audinat E (1999) Postsynaptic glutamate receptors and integrative properties of fast-spiking interneurons in the rat neocortex. J Neurophysiol 82:1295-1302.

Armstrong-James M, Welker E, Callahan CA (1993) The contribution of NMDA and non-NMDA receptors to fast and slow transmission of sensory information in the rat SI barrel cortex. J Neurosci $13: 2149-2160$

Beaulieu C (1993) Numerical data on neocortical neurons in adult rat, with special reference to the GABA population. Brain Res 609:284-292.

Benshalom G, White EL (1986) Quantification of thalamocortical synapses with spiny stellate neurons in layer IV of mouse somatosensory cortex. J Comp Neurol 253:303-314.

Bernardo KL, Woolsey TA (1987) Axonal trajectories between mouse somatosensory thalamus and cortex. J Comp Neurol 258:542-564.

Bi G, Poo M (1998) Synaptic modifications in cultured hippocampal neurons: dependence on spike timing, synaptic strength, and postsynaptic cell type. J Neurosci 18:10464-10472.

Brumberg JC, Pinto D, Simons DJ (1996) Spatial gradients and inhibitory summation in the rat whisker barrel system. J Neurophysiol 76:130-140.

Brumberg JC, Pinto DJ, Simons DJ (1999) Cortical columnar processing in the rat whisker-to-barrel system. J Neurophysiol 82:1808-1817.

Davison AC, Hinkley DV (1997) Bootstrap methods and their application. Cambridge, UK: Cambridge UP.

Efron B, Tibshirani R (1991) Statistical data analysis in the computer age. Science 253:390-395.

Ferster D, Chung S, Wheat H (1996) Orientation selectivity of thalamic input to simple cells of cat visual cortex. Nature 380:249-252.

Gibson JR, Beierlein M, Connors BW (1999) Two networks of electrically coupled inhibitory neurons in neocortex. Nature 402:75-79.

Goldreich D, Kyriazi HT, Simons DJ (1999) Functional independence of layer IV barrels in rodent somatosensory cortex. J Neurophysiol 82:1311-1316.

Gray CM, McCormick DA (1996) Chattering cells: superficial pyramidal neurons contributing to the generation of synchronous oscillations in the visual cortex. Science 274:109-113.

Hamilton LC (1991) Regression with graphics. Belmont, CA: Duxbury.

Hartings JA, Temereanca S, Simons DJ (2000) High responsiveness and direction sensitivity of neurons in the rat thalamic reticular nucleus to vibrissal deflections. J Neurophysiol 83:2791.

Hubel DH, Wiesel TN (1962) Receptive fields, binocular interaction, and functional architecture in the cat's visual cortex. J Physiol (Lond) 160:106-154.

Johnson MJ, Alloway KD (1996) Cross-correlation analysis reveals laminar differences in thalamocortical interactions in the somatosensory system. J Neurophysiol 75:1444-1457.

Kawaguchi Y (1993) Groupings of nonpyramidal and pyramidal cells with specific physiological and morphological characteristics in rat frontal cortex. J Neurophysiol 69:416-431.

Kawaguchi Y, Kubota Y (1993) Correlation of physiological subgroupings of nonpyramidal cells with parvalbumin- and calbindinD28kimmunoreactive neurons in layer $\mathrm{V}$ of rat frontal cortex. J Neurophysiol 70:387-396.

Keller A, Carlson GC (1999) Neonatal whisker clipping alters intracortical, but not thalamocortical projections, in rat barrel cortex. J Comp Neurol 412:83-94.

Keller A, White EL (1987) Synaptic organization of GABAergic neurons in mouse SmI cortex. J Comp Neurol 262:1-12.

Kyriazi HT, Simons DJ (1993) Thalamocortical response transformations in simulated whisker barrels. J Neurosci 13:1601-1615.

Kyriazi HT, Carvell GE, Simons DJ (1994) OFF response transformations in the whisker/barrel system. J Neurophysiol 72:392-401.

Kyriazi HT, Carvell GE, Brumberg JC, Simons DJ (1996) Quantitative effects of GABA and bicuculline methiodide on receptive field properties of neurons in real and simulated whisker barrels. J Neurophysiol 75:547-560.

Land PW, Buffer Jr SA, Yaskosky JD (1995) Barreloids in adult rat thalamus: three dimensional architecture and relationship to somatosensory cortical barrels. J Comp Neurol 355:573-588.

Levick W R, Cleland BG, Dubin MW (1972) Lateral geniculate neurons of cat: retinal inputs and physiology. Invest Ophthalmol 11:302-311.

McCasland JS, Carvell GE, Simons DJ, Woolsey TA (1991) Functional asymmetries in the rodent barrel cortex. Somatosens Mot Res 8:111-116.

McCormick DA, Connors BW, Lighthall JW, Prince DA (1985) Comparative electrophysiology of pyramidal and sparsely spiny stellate neurons of the neocortex. J Neurophysiol 54:782-806.

Miller LM, Escabi MA, Read HL, Schreiner CE (2001) Functional convergence of response properties in the auditory thalamocortical system. Neuron 32:151-160.

Moore DS, McCabe GP (1993) Introduction to the practice of statistics. New York: Freeman.

Mountcastle VB, Talbot WH, Sakata H, Hyvarinen J (1969) Cortical neuronal mechanisms in flutter-vibration studied in unanesthetized monkeys: neuronal periodicity and frequency discrimination. J Neurophysiol 32:452-484.

Perkel DH, Gerstein GL, Moore GP (1967) Neuronal spike trains and stochastic point processes. II. Simultaneous spike trains. Biophys J 7:419-440.

Pinto DJ, Brumberg JC, Simons DJ, Ermentrout GB (1996) A quantitative population model of whisker barrels: re-examining the WilsonCowan equations. J Comp Neurosci 3:247-264.

Pinto D, Brumberg JC, Simons D (2000) Circuit dynamics and coding strategies in rodent somatosensory cortex. J Neurophysiol 83:1158-1166.

Pinto DJ, Hartings JA, Brumberg JC, Simons DJ (2002) Cortical damping: analysis of thalamocortical response transformations in rodent barrel cortex. Cereb Cortex, in press.

Porter JT, Johnson CK, Agmon A (2001) Diverse types of interneurons generate thalamus-evoked feedforward inhibition in the mouse barrel cortex. J Neurosci 21:2699-2710. 
Reid CR, Alonso J-M (1995) Specificity of monosynaptic connections from thalamus to visual cortex. Nature 378:281-284.

Rudy B, Chow A, Lau D, Amarillo Y, Ozaita A, Saganich M, Moreno H, Nadal MS, Hernandez-Pineda R, Hernandez-Cruz A, Erisir A, Leonard C, Vega-Saenz de Miera E (1999) Contributions of Kv3 channels to neuronal excitability. Ann NY Acad Sci 868:304-343.

Simons DJ (1978) Response properties of vibrissa units in the rat SI somatosensory neocortex. J Neurophysiol 41:798-820.

Simons DJ (1983) Multi-whisker stimulation and its effects on vibrissa units in rat SmI barrel cortex. Brain Res 276:178-182.

Simons DJ, Carvell GE (1989) Thalamocortical response transformation in the rat vibrissa/barrel system. J Neurophysiol 61:311-330.

Simons DJ, Land PW (1987) Early experience of tactile stimulation influences organization of somatic sensory cortex. Nature 326:694-697.

Simons DJ, Woolsey TA (1984) Morphology of Golgi-Cox-impregnated barrel neurons in rat SmI cortex. J Comp Neurol 230:119-132.

Swadlow HA (1995) Influence of VPM afferents on putative inhibitory interneurons in SI of the awake rabbit: evidence from cross-correlation, microstimulation, and latencies to peripheral sensory stimulation. J Neurophysiol 73:1584-1599.

Swadlow HA, Gusev AG (2002) Receptive-field construction in cortical inhibitory interneurons. Nat Neurosci 5:403-404.

Tanaka K (1985) Organization of geniculate inputs to visual cortical cells in the cat. Vis Res 25:357-364.

Welker C, Woolsey TA (1974) Structure of layer IV in the somatosensory neocortex of the rat: description and comparison with the mouse. J Comp Neurol 158:437-453.

White EL (1978) Identified neurons in mouse SmI cortex which are postsynaptic to thalamocortical axon terminals: a combined golgi-electron microscopic and degeneration study. J Comp Neurol 181:627-661.

White EL, Benshalom G, Hersch SM (1984) Thalamocortical and other synapses involving nonspiny multipolar cells of mouse SmI cortex. J Comp Neurol 229:311-320.

Woolsey TA, Dierker ML, Wann DF (1975) Mouse SmI cortex: qualitative and quantitative classification of Golgi-impregnated barrel neurons. Proc Natl Acad Sci USA 72:2165-2169. 